\title{
Modelling of Melt Pressure Development in Polymer Extrusion: Effects of Process Settings and Screw Geometry
}

\author{
Document Version \\ Accepted author manuscript
}

Link to publication record in Manchester Research Explorer

Citation for published version (APA):

Abeykoon, C., Li, K., Martin, P. J., \& Kelly, A. L. (2011). Modelling of Melt Pressure Development in Polymer Extrusion: Effects of Process Settings and Screw Geometry. In host publication (pp. 197-202)

\section{Published in:}

host publication

\section{Citing this paper}

Please note that where the full-text provided on Manchester Research Explorer is the Author Accepted Manuscript or Proof version this may differ from the final Published version. If citing, it is advised that you check and use the publisher's definitive version.

\section{General rights}

Copyright and moral rights for the publications made accessible in the Research Explorer are retained by the authors and/or other copyright owners and it is a condition of accessing publications that users recognise and abide by the legal requirements associated with these rights.

\section{Takedown policy}

If you believe that this document breaches copyright please refer to the University of Manchester's Takedown Procedures [http://man.ac.uk/04Y6Bo] or contact uml.scholarlycommunications@manchester.ac.uk providing relevant details, so we can investigate your claim.

\section{OPEN ACCESS}




\title{
Modelling of Melt Pressure Development in Polymer Extrusion: Effects of Process Settings and Screw Geometry
}

\author{
Chamil Abeykoon, Kang Li, Peter J. Martin and Adrian L. Kelly
}

\begin{abstract}
Melt pressure is one of the most important process parameters in polymer extrusion and it is also closely related to the product quality. However, it is not directly controllable and is affected in a complex manner by changing other process operating conditions such as screw rotation speed and set temperatures. The ability to predict such parameter would be a powerful tool to aid process design and optimisation. However, only a few practical process models are available to predict the melt pressure based on process settings in polymer extrusion. This paper describes new nonlinear static and linear dynamic models which have been developed to explore the effects of process settings and screw geometry on melt pressure development in single screw extrusion. A computationally efficient linear-in-the-parameters modelling technique was used in model development and the resultant models show satisfactory performance in predicting the melt pressure with good accuracy over a wide operating window.
\end{abstract}

\section{INTRODUCTION}

Melt pressure is an important characteristic of single screw extruders and influences to the process output [1]. In most extrusion processes, it is common to measure pressure at the end of the extruder barrel and/or at the die. Sometimes, extruders are instrumented with multiple pressure transducers which are placed along the length of the extruder barrel. Generally, barrel pressure information helps the understanding of the processing behaviour and is useful in troubleshooting [2]. Moreover, melt pressure is dependent on process settings, machine geometry, and material properties [3]. However, investigation of the effects of process settings on melt pressure development is important for a given machine, screw geometry, and polymer. Attempts made previously on modelling melt pressure in single screw extrusion are discussed in the following sections.

Donovan [4] made an analytical approach to predict the pressure profile in the melting (or compression) and metering zones of a single screw extruder. It was found that the pressure profile is highly dependent on the solid bed of polymer under stable melting conditions. Lovegrove and Williams [5] proposed a theoretical analysis to predict the pressure generation in the feed zone of single screw extruders and emphasised the importance of considering screw weight and centrifugal forces for pressure calculations. Lindt [1] found that increasing throughput tends to decrease the pressure

C. Abeykoon and P. J. Martin are with the School of Mechanical and Aerospace Engineering, Queen's University Belfast, Belfast, BT9 5AH, UK E-mail: yabeykoon01@qub.ac.uk (Chamil Abeykoon)

$\mathrm{K} . \mathrm{Li}$ is with the School of Electronics, Electrical Engineering and Computer Science, Queen's University Belfast, Belfast, BT9 5AH, UK

A. L. Kelly is with the IRC in Polymer Science and Technology, School of Engineering, Design and Technology, University of Bradford, Bradford, BD7 1DP, UK build up capacity of the melting zone in a single screw extruder. Moreover, a relationship between the melting mechanism and pressure profile was discussed. Work presented by Potente and Jungemann [6] developed polynomial formulae to describe the correlation between axial pressure profile and mass throughput along the melting zone which can be used as simulation program models. These models are only applicable for non-Newtonian polymer melts and the authors found good agreement with numerical results tested.

Rauwendaal [7] developed a formula to describe throughput as a function of the pressure gradient for power law fluids in single screw extruders and mentioned that the expression was well matched with numerical calculations carried out. Furthermore, he argued that when extruder die pressure changes with time, the process output may vary and hence dimensional instability of the products can be caused [8]. Another study by Rauwendaal [9] developed a relationship between extruder output and pressure and found that at low melt flow index values, pressure sensitivity of the output increased as the output reduced. Moreover, a comprehensive analysis of extrusion pressure was provided and proposed equations to calculate axial and circumferential pressure profiles and the usefulness of process information given by these pressure profiles was also highlighted [2].

Work presented by Tadmor et al. [10] considered the transport momentum in the solid bed, melt film, and melt pool of a single screw extruder. Equations were developed to represent the dynamic pressure profile through simulations. A number of assumptions were made and the model involved complex differential equations requiring powerful computer solutions. As a result, these models could not be applied in practical applications. Work presented by Costin et al. [11] used time series analysis to develop dynamic transfer function models between the screw speed and pressure and also to model the disturbances associated with the extruder pressure. Kochhar and Parnaby et al. [12] proposed two time series models of melt pressure and temperature. Linearised models relating to the small perturbations of the screw speed were identified by observing corresponding changes of the melt pressure and the temperature at the die and the model was used in formulating a process control framework. Tan et al. [13] proposed a grey-box model of polymer extrusion and stated that the model can be used to estimate melt temperature and melt pressure at the die. The model was verified through simulation by introducing step changes to the parameters manipulated such as the screw speed and it was reported that the model adopted to the changes of processing conditions and feed materials. 
Botten et al. [14] proposed a theoretical model to predict the melt pressure in a single screw extruder as a function of machine geometry (i.e. barrel diameter, screw helix angle and channel width), material properties (i.e. yield and shear stresses), screw speed and volumetric flow rate. Experimental work is underway to verify the model performance. Bereaux et al. [15] introduced a model to predict the throughput and melt pressure in single screw extrusion and found that the throughput is primarily dependent upon screw geometry while the peak pressure is highly dependent on polymer viscosity. Also, it was argued that the level of back pressure (i.e. pressure close to the die entry) in polymer extrusion is dependent upon the screw and die geometries.

From the literature, it is clear that little reported work is available on modelling melt pressure in polymer extrusion based on system identification experiments. Obviously, pressure fluctuations may cause problems in product quality and these fluctuations provide a good indication of process functional quality. Proper observation of the melt pressure behaviour of the process is essential otherwise excessive pressure generation may lead to catastrophic failures [8]. Perhaps, existing theoretical models may not provide the actual process dynamics due to a number of simplifying assumptions. Therefore, understanding the actual effects of process settings (i.e. screw speed and barrel set temperatures), materials, and machine geometry on pressure development are highly important to maintain the process stability while ensuring process safety.

In this work, nonlinear static and linear dynamic models are proposed to predict the extruder melt pressure development with two different screw geometries. The effects on melt pressure development of each process parameter and the screw geometry are also discussed. This study was focused on a single screw extruder with one material.

\section{EQUIPMENT AND PROCEDURE}

All measurements were carried out on a $63.5 \mathrm{~mm}$ diameter (D) single screw extruder (Davis Standard BC-60).

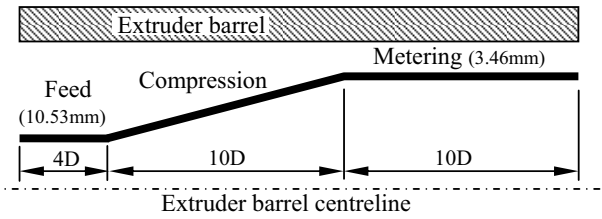

(a)

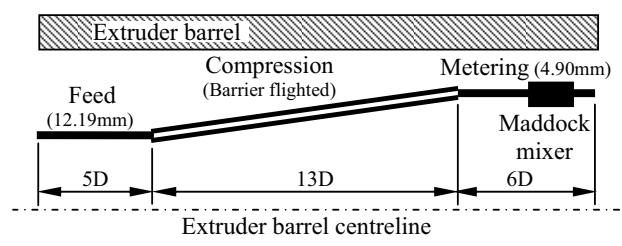

(b)

Fig. 1. Details of the screws: (a). The GC screw (b). The BF screw

A barrier flighted screw with a spiral Maddock mixer (DSB1 general purpose screw with a 2.5:1 compression ratio) and a tapered gradual compression screw (with 3:1 compression ratio) were used to process material and details are shown in Figure 1. From here onwards, these two screws are denoted as the BF screw and the GC screw respectively. The extruder was fitted with a $38 \mathrm{~mm}$ diameter adapter by using a clamp ring prior to a short $6 \mathrm{~mm}$ diameter capillary die as shown in Figure 2.

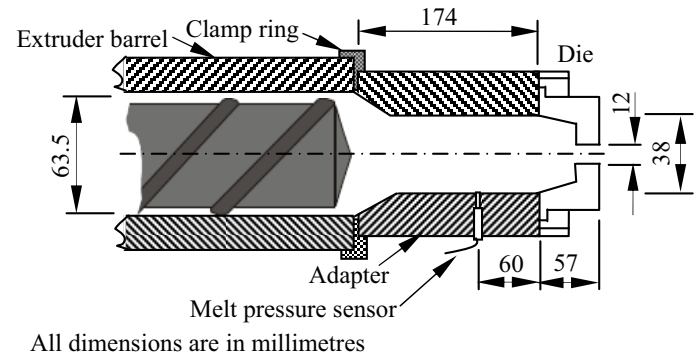

Fig. 2. Extruder die, adapter, and pressure sensor

The extruder barrel has four separate temperature zones and another three separate temperature zones at the clamp ring, adapter and die. All of these temperature zones are equipped with Davis Standard Dual Therm controllers. The extruder barrel dimensions and the arrangement of the heaters are shown in Figure 3.

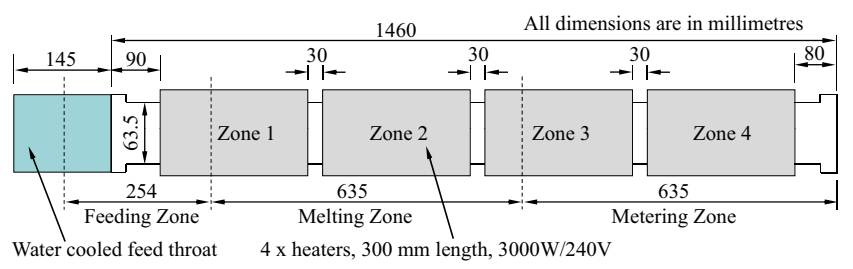

Fig. 3. Arrangement of the Betol BC-60 extruder barrel and heaters

Melt pressure at the adapter (i.e. close to the die entry) was measured using a PT422A Dynisco pressure sensor as shown in Figure 2. A LabVIEW software program was developed to communicate between the experimental instruments and a PC. The screw speed, barrel temperatures and all melt pressure signals were acquired at $10 \mathrm{~Hz}$ using a 16-bit DAQ card (National Instruments (NI) PCI-6035E) through a NI low-noise SCXI-1000 connector box.

Experimental trials were carried out on a virgin high density polyethylene (HDPE), (ExxonMobil HYA 800), (density: $0.961 \mathrm{~g} / \mathrm{cm}^{3}$, melt flow index (MFI): 0.7g/10min @ $\left(190^{\circ} \mathrm{C}\right.$, $2.16 \mathrm{~kg})$ ). The extruder temperature settings were fixed as described in Table I under three different barrel set temperature conditions and denoted as A (high temperature), B (medium temperature), and C (low temperature).

TABLE I

EXTRUDER BARREL TEMPERATURE SETTINGS

\begin{tabular}{|c|c|c|c|c|c|c|c|}
\hline \multirow{3}{*}{$\begin{array}{c}\text { Temperature } \\
\text { settings }\end{array}$} & \multicolumn{7}{|c|}{ Set temperatures $\left({ }^{\circ} \mathrm{C}\right)$} \\
\hline & \multicolumn{4}{|c|}{ Barrel zones } & \multirow{2}{*}{ Clamp ring } & \multirow{2}{*}{ Adapter } & \multirow{2}{*}{ Die } \\
\hline & 1 & 2 & 3 & 4 & & & \\
\hline $\mathrm{A}$ & 110 & 130 & 180 & 230 & 230 & 230 & 230 \\
\hline $\mathrm{B}$ & 105 & 125 & 175 & 215 & 215 & 215 & 215 \\
\hline C & 100 & 120 & 170 & 200 & 200 & 200 & 200 \\
\hline
\end{tabular}


Experiments were started with temperature setting A and data was recorded with the screw stationary for 1 minute. Then, the screw speed was increased up to 90rpm with random steps of between \pm 5 and 40rpm and for the different barrel set temperatures with the extruder running for about 151 and 193 minutes continuously in the case of the GC screw and BF screw respectively. The extruder was allowed to stabilise over 15 minutes after each set temperature change whereas it was running for about 7 minutes over each of the other different conditions. All of these settings were selected in order to generate realistic processing conditions whilst covering the full operating range of the extruder (i.e. 0-100rpm). Separate tests were carried out for model training and validation.

\section{MODELLING}

In general, the melt pressure development $(P)$ in polymer extrusion can be represented as a function of $\omega_{s c}$ and $T_{b}$ :

$$
P=f\left(\omega_{s c}, T_{b}\right)
$$

where $\omega_{s c}$ is the screw speed and $T_{b}$ represents the barrel set temperatures (subscript $b$ represents the different barrel zones $\left.T_{1}-T_{4}\right)$. Five model inputs $\left(\omega_{s c}, T_{1}, T_{2}, T_{3}\right.$, and $\left.T_{4}\right)$ and one output $(P)$ were considered for the modelling of melt pressure generated as a function of process speed and barrel set temperatures. The selected model structure is illustrated in Figure 4.

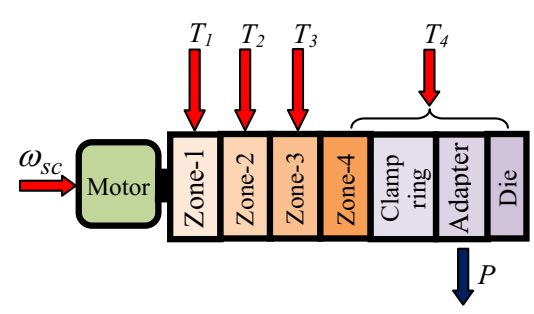

Fig. 4. Model structure with the selected inputs and output

The set temperatures of the clamp ring, the adapter, and the die were always equal to $T_{4}$ in this study. If these values vary from $T_{4}$, it is possible to add them as three different model inputs. There are 19 and 25 different processing situations and each input signal used for the model training contains 90,600 and 115,800 data points for the GC and the BF screws respectively. The model inputs matrices for both training and validation data are shown in Figures 5 and 6 for the two screws. The model output contains the measured melt pressure at each processing condition corresponding to the relevant inputs and the signal length is same as the input signal length.

In this work, a linear-in-the-parameters (LITP) modelling technique was used to model the extrusion process. In general, a LITP model is a linear combination of model terms which are linear or nonlinear functions of the corresponding system variables and it gives the target output as a linear combination of model terms, the coefficient of each model term can be estimated by linear optimisation methods, such as least squares. This is a common way to build a nonlinear system model as the linear systems are too simple

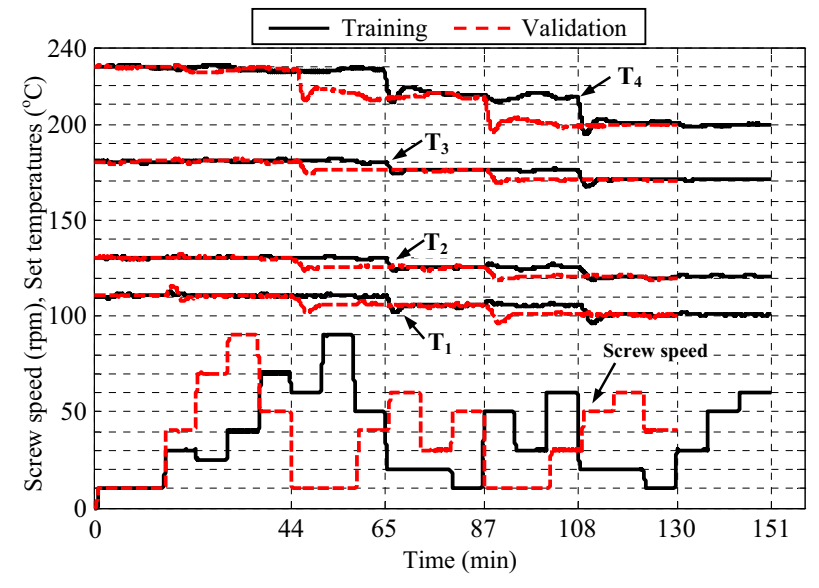

Fig. 5. Model inputs matrices - GC screw

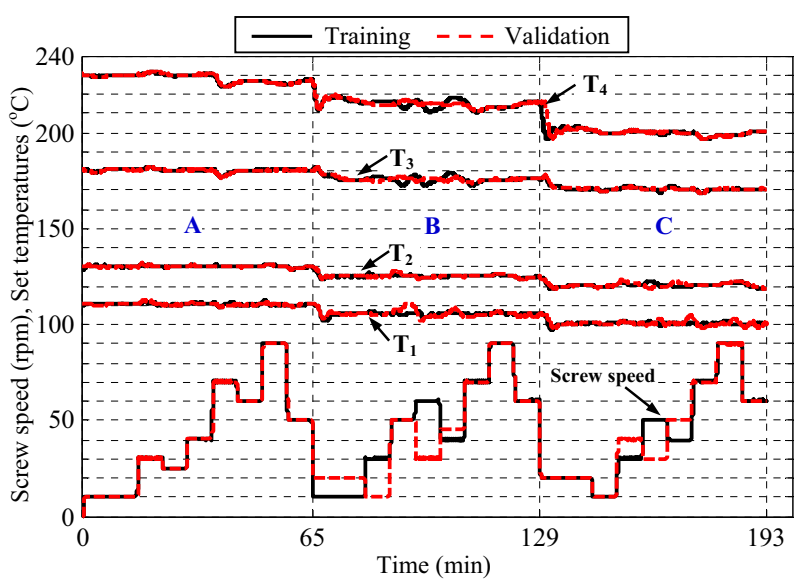

Fig. 6. Model inputs matrices - BF screw

to approximate the nonlinear characteristics and traditional nonlinear systems are difficult to optimise [16], [17]. Generally, most of the nonlinear optimisation methods require extensive computation and a large amount of memory and the resulting model is usually a sub-optimal. Therefore, the LITP model is a compromise of the above two construction schemes as it does not require huge computational power for structure and parameter estimations. Due to their ability for universal approximation and simple structure, LITP models have been widely used in a number of practical applications such as signal processing, data mining, pattern recognition, time series prediction, and nonlinear system modelling and identification etc [18], [19], [20].

A two-stage algorithm [16], [17] was employed in the selection and refinement of the LITP models for this study. In the first stage, a fast recursive algorithm (FRA) was used for the selection of the model structure and for estimation of the model parameters. This solves the problem recursively and does not require matrix decomposition as is the case for orthogonal least squares (OLS) techniques [21]. However, the models developed include a constraint that the terms added later are based on previously selected ones. As a result, some of them may not have a significant contribution. Then, in the 
second stage a backward model refinement procedure was carried out to eliminate non-significant terms to build up a compact model. The significance of each selected model term was reviewed and compared with those remaining in the candidate term pool and all insignificant terms were replaced, leading to improved performance without increasing the model size. The authors have used the same modelling technique for the modelling of the die melt temperature profile in polymer extrusion and good results have been achieved [22].

\section{RESUlTS AND Discussion}

Firstly, linear models were adopted to approximate the system under static and dynamic conditions. However, performance of the linear models under static conditions were poorer and hence static nonlinear models were developed. Linear dynamic models were able to provide good performance in predicting melt pressure development of both screw geometries. Therefore, the static nonlinear and dynamic linear models are discussed in detail. To test the dynamic model accuracy, the modelling errors (ME) and the root mean square errors (RMSE) of the models were determined by equations (2) and (3) respectively.

$$
\begin{gathered}
M E=y_{i}(t)-\hat{y}_{i}(t) \\
R M S E=\sqrt{\frac{1}{N} \sum_{i=1}^{N}\left[\left(\hat{y}_{i}(t)-y_{i}(t)\right)\right]^{2}}
\end{gathered}
$$

where $y_{i}(t)$ is the measured melt pressure at time $t, \hat{y}_{i}(t)$ is the model estimated melt pressure at time $t$, and $N$ is the number of data points. The same equations without the involvement of time were used to test the accuracy of static models.

1) Linear dynamic models: For the selection of the linear dynamic melt pressure prediction model, a number of different model combinations (i.e. models with different orders and number of terms) were studied. Two past output terms and one past input term from each input were used to predict the current output (i.e. $n_{a}=2$ and $n_{b}$ for each input is equal to $1)$. Then the maximum delays $\left(n_{k}\right)$ attributed to each model input had to be determined. Therefore, melt pressure changes followed by screw speed changes and barrel set temperature changes were observed from the experimentally measured data. The process pressure changes immediately after any change of screw speed. Also, the melt pressure is affected by barrel set temperatures but it takes a slightly longer period of time to change the barrel zone temperatures once any is change made. Based on these observations reasonable values were assumed for the delays attributed to each input as: $d_{\omega_{s c}}=10 \mathrm{~s}, d_{T 1}=120 \mathrm{~s}, d_{T 2}=120 \mathrm{~s}, d_{T 3}=120 \mathrm{~s}$, and $d_{T 4}=120 \mathrm{~s}$. These delays can be adjusted as required depending on the screw geometry, material, processing conditions etc. The details of some of the dynamic models studied are shown in Table II along with their root mean square errors when compared with the unseen data for both screws.
TABLE II

RMSE (ON THE UNSEEN DATA) OF THE STUDIED DYNAMIC MODELS WITH DIFFERENT ORDERS AND NUMBER OF TERMS

\begin{tabular}{|c|c|c|c|c|c|c|}
\hline \multirow{2}{*}{\begin{tabular}{c} 
Number $\begin{array}{c}\text { of } \\
\text { terms }\end{array}$ \\
\cline { 2 - 7 }
\end{tabular}} & \multicolumn{3}{|c|}{ GC screw with the selected model order } \\
\hline & 1 & 2 & 3 & 1 & 2 & 3 \\
\hline 1 & 0.0127 & 0.0127 & 0.0127 & 0.0333 & 0.0333 & 0.0333 \\
\hline 2 & 0.0126 & 0.0126 & 0.0126 & 0.0328 & 0.0328 & 0.0328 \\
\hline 3 & 0.0126 & 0.0126 & 0.0126 & 0.0328 & 0.0328 & 0.0328 \\
\hline 4 & 0.0126 & 0.0126 & 0.0126 & 0.0328 & 0.0328 & 0.0328 \\
\hline 5 & 0.0126 & 0.0126 & 0.0126 & 0.0328 & 0.0322 & 0.0322 \\
\hline 6 & 0.0126 & 0.0129 & 0.0129 & 0.0328 & 0.0316 & 0.0317 \\
\hline 7 & $\mathbf{0 . 0 1 2 6}$ & 0.0133 & 0.0133 & $\mathbf{0 . 0 3 2 8}$ & 0.0316 & 0.0314 \\
\hline 8 & 0.0126 & 0.0133 & 0.0133 & 0.0328 & 0.0311 & 0.0314 \\
\hline
\end{tabular}

From the linear dynamic models studied, models with 7 terms were selected for further analysis and are shown in
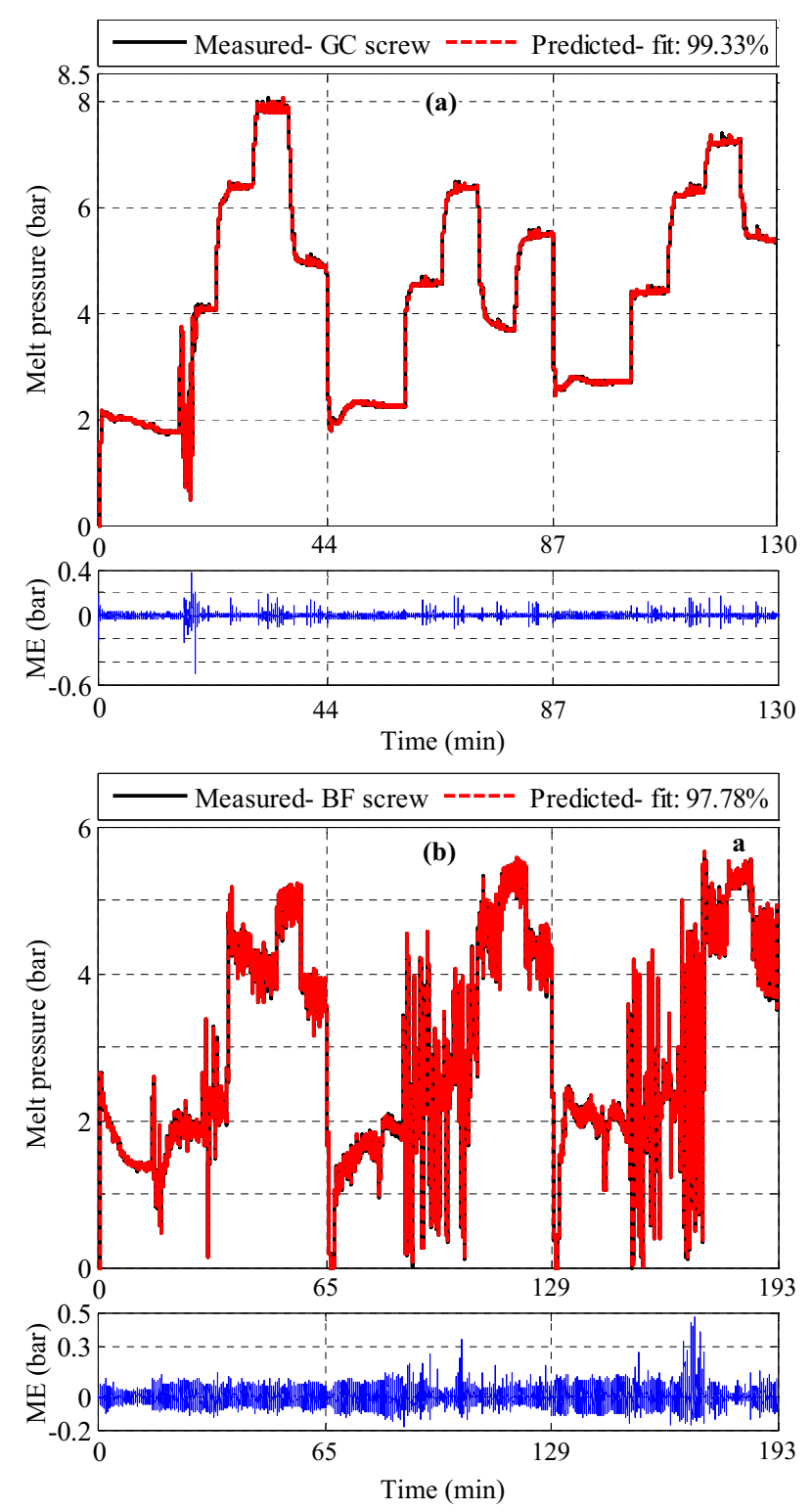

Fig. 7. Performance of the selected linear dynamic models on the unseen data with the model prediction error (ME): (a). GC screw (b). BF screw 
equations (4) and (5) for the GC and BF screws respectively. These models show root mean square errors of 0.013 and 0.033 on the unseen data respectively.

$$
\begin{aligned}
& \hat{P}_{G C}(t)=0.65331 \times \hat{P}_{G C}(t-1)+0.34513 \times \hat{P}_{G C}(t-2) \\
& +0.00031 \times T_{3}(t-120) \quad-0.00019 \times T_{2}(t-120) \\
& +0.00013 \times \omega_{s c}(t-10)-0.00010 \times T_{4}(t-120) \\
& -6.18332 \times 10^{-05} \times T_{1}(t-120) \\
& \hat{P}_{B F}(t)=0.89464 \times \hat{P}_{B F}(t-1)+0.10459 \times \hat{P}_{B F}(t-2) \\
& -0.00022 \times T_{2}(t-120)-7.43988 \times 10^{-05} \times T_{1}(t-120) \\
& +0.00014 \times T_{3}(t-120)+5.38979 \times 10^{-05} \times T_{4}(t-120) \\
& +4.69300 \times 10^{-05} \times \omega_{s c}(t-10)
\end{aligned}
$$

The prediction ability of the selected dynamic models on the unseen data is shown in Figure 7 for both screws along with their model prediction errors. In general, both models show good prediction accuracy on the unseen data over all the processing conditions.

2) Nonlinear static models: For the selection of static models different model combinations were also studied and details are given in Table III along with their root mean square errors on the unseen data for both screws.

TABLE III

RMSE (ON THE UNSEEN DATA) OF THE STUDIED STATIC MODELS WITH DIFFERENT ORDERS AND NUMBER OF TERMS

\begin{tabular}{|c|c|c|c|c|c|c|}
\hline \multirow{2}{*}{\begin{tabular}{c} 
Number $\begin{array}{c}\text { of } \\
\text { terms }\end{array}$ \\
\cline { 2 - 7 }
\end{tabular}} & \multicolumn{3}{|c}{ GC screw with the selected model order } \\
\hline & 1 & 2 & 3 & 1 & 2 & 3 \\
\hline 1 & 1.0701 & 1.0701 & 1.0701 & 0.8883 & 0.8883 & 0.8883 \\
\hline 2 & 0.0688 & 0.7928 & 0.7885 & 0.8092 & 0.8092 & 0.8092 \\
\hline 3 & 0.4170 & 0.4224 & 0.4240 & 0.8099 & 0.8038 & 0.8117 \\
\hline 4 & 0.3967 & 0.4135 & 0.3095 & 0.8117 & 0.7776 & 0.7917 \\
\hline 5 & 0.3996 & 0.4112 & 0.4029 & 0.8137 & 0.7737 & 0.8009 \\
\hline 6 & 0.3996 & 0.4069 & 0.3990 & 0.8137 & 0.7812 & 0.7783 \\
\hline 7 & 0.3996 & 0.4079 & 0.4045 & 0.8137 & 0.7761 & 0.7679 \\
\hline 8 & 0.3996 & 0.4129 & 0.4057 & 0.8137 & 0.7752 & 0.7630 \\
\hline 9 & 0.3996 & 0.4087 & 0.4121 & 0.8137 & 0.7734 & 0.7552 \\
\hline 10 & 0.3996 & 0.4095 & 0.4087 & 0.8137 & 0.7733 & 0.7617 \\
\hline 11 & 0.3996 & 0.4238 & 0.4145 & 0.8137 & 0.7899 & 0.7520 \\
\hline 12 & 0.3996 & $\mathbf{0 . 3 9 8 1}$ & 0.4258 & 0.8137 & $\mathbf{0 . 7 9 0 8}$ & 0.7551 \\
\hline 13 & 0.3996 & 0.4098 & 0.4049 & 0.8137 & 0.7926 & 0.7556 \\
\hline 14 & 0.3996 & 0.4036 & 0.4279 & 0.8137 & 0.7922 & 0.7560 \\
\hline
\end{tabular}

Finally $2^{\text {nd }}$ order static nonlinear models with 12 terms were selected for discussion and are given in equations (6) and (7) for the GC and BF screws respectively.

$$
\begin{aligned}
P_{G C}= & -0.42177 \times \omega_{s c}+0.21133 \times T_{3} \quad-0.05896 \times T_{2} \times T_{3} \\
& +0.03528 \times T_{2}^{2}+0.00075 \times T_{1} \times T_{4}+0.00920 \times \omega_{s c} \times T_{3} \\
& -0.00237 \times T_{4}^{2}-0.00498 \times \omega_{s c} \times T_{1}-0.00458 \times \omega_{s c} \times T_{2} \\
& -0.00014 \times \omega_{s c}^{2}+0.00733 \times T_{2} \times T_{4}+0.01953 \times T_{3}^{2} \quad(6) \\
P_{B F}= & +2.70469 \times T_{3}-1.43535 \times T_{4} \quad-1.33292 \times T_{2} \\
& +0.89517 \times \omega_{s c}+0.09102 \times T_{2} \times T_{3}-0.08794 \times T_{2}^{2} \\
& -0.03979 \times T_{3}^{2}+0.03357 \times T_{2} \times T_{4}-0.00887 \times \omega_{s c} \times T_{3} \\
& -0.00643 \times T_{4}^{2}+0.00312 \times \omega_{s c} \times T_{4}+0.00052 \times \omega_{s c}^{2} \quad(7)
\end{aligned}
$$

These polynomial static models of the GC and BF screws show 0.398 and 0.791 root mean square errors on the unseen data respectively.
3) Effects of process settings and screw geometry on melt pressure: As shown in section II, both experiments were carried out on the same material and under the same processing conditions (i.e. the same barrel set temperatures). As evidenced by Figure 7, a relatively stable pressure generation can be seen with the GC screw, whereas experiments with the BF screw show pressure fluctuations over some screw speeds. According to Gitschner et al. [23], process pressure variations are inter-related with the barrel zone temperatures and process cooling systems and it is possible to stabilise pressure surges by adjusting these parameters. In this study, it was felt that fluctuations observed with the BF screw were as a result of the incompatibility of the selected barrel set temperatures with the screw geometry. It was very difficult to select suitable barrel set temperatures for the HDPE material used as the material tended to slip on the screw. As a result material conveying was poor and gave very low process pressures and hence reduced throughput rates. Therefore, the barrel set temperatures shown in Table I were selected after long periods of adjustments, particularly in the first two barrel zones (i.e. $T_{1}$ and $T_{2}$ ) to avoid conveying problems. These adjustments were only carried out with the GC screw and then the experiments with the BF screw were carried out with the same set temperatures. This may be the reason for having higher pressure fluctuations with the BF screw than the GC screw as shown in Figure 7. Usually, BF screws perform favourably (e.g. efficient melting and mixing) compared to conventional GC screws [24], [25]. Thus, the results in this study support the argument made by Gitschner that barrel set temperatures have to be changed from screw to screw although other test conditions remain constant. However, this material slipping condition may depend on the frictional and shear forces in-between the metal surfaces (i.e. barrel and screw) and the pellets (i.e. material) and also on the pellet size and shape [8], [26]. Achieving proper material conveying (i.e. a proper axial pressure profile) along all screw zones is a major requirement to achieve stable pressure generation and this ensures a uniform process output [2]. Thus, appropriate set temperatures should be selected for all barrel temperature zones to avoid possible conveying problems which may lead to pressure fluctuations.

In general, it can be considered that the experiments were carried out under proper material conveying conditions. Therefore, the models developed can be used to identify the effects of process settings and screw geometry on melt pressure development. As shown in Figure 7, the process pressure development is higher with the GC screw than the BF screw, which can be attributed to the geometrical differences between the screws. According to the static models, screw speed is the most significant process parameter for the GC screw while the third barrel zone temperature $\left(T_{3}\right)$ is the most significant with the BF screw. Of the barrel zone temperatures, zone 3 temperature seems to be the most significant for both experiments under static processing conditions despite the differences in screw geometry. As shown in Figure 3, $T_{3}$ pertains both the melting and metering zones of the extruder barrel. In the dynamic models, all barrel set temperatures 
and screw speed show low coefficients. Overall, all static and dynamic models show the significance of the end barrel zones temperatures (i.e. $T_{2}, T_{3}$, and $T_{4}$ ) on melt pressure development where molten material exists. Therefore, under proper conveying conditions the significance of the feed (or solids conveying) zone temperature $\left(T_{1}\right)$ on the process melt pressure seems to be negligible.

\section{CONCLUSIONS AND FUTURE WORKS}

\section{A. Conclusions}

New static and dynamic models were presented to predict the melt pressure in polymer extrusion as a function of readily measurable process parameters. A computationally efficient LITP modelling technique was used and the models developed were able to predict the process melt pressure with good accuracy over a wide operating window. These models were used to identify the effects on the melt pressure of individual processing parameters and screw geometry in the process. The results show that the process settings should be selected based on the screw geometry for the different materials to avoid problematic conveying issues which may lead to pressure fluctuations. Moreover, the significance of each processing parameter on the level of melt pressure seems to be dependent upon the screw geometry. Also, the predictions from the proposed models are in agreement with the measured processing conditions and hence they are useful in processing issues such as obtaining optimum process settings to avoid possible melt pressure fluctuations.

\section{B. Future Works}

This study was carried out on a single material with two different screw geometries and one die geometry. Development of models for different materials and die geometries will help to identify the specific effects of material and die geometry on melt pressure development. Moreover, melt pressure is not only a function of process settings and consideration of both the effects of material properties, (e.g. thermal conductivity, melt consistency index, etc), and machine geometry, (e.g. screw channel depth, barrel diameter, surface conditions of the barrel and screw, barrel thickness), should help to improve the model performance further and will be examined in the future work. Also, the models will be used to establish the optimum process settings to minimise melt pressure fluctuations.

\section{ACKNOWLEDGMENTS}

This work was supported by Engineering and Physical Sciences Research Council (EPSRC) under Grant number EP/F021070/1. The authors would like to thank all who supported the research from Queen's University Belfast and University of Bradford.

\section{REFERENCES}

[1] J. T. Lindt, "Pressure development in the melting zone of a singlescrew extruder," Polymer Engineering and Science, vol. 16, no. 4, pp. 1162-1166, 1981.

[2] C. Rauwendaal, "Pressure distribution in screw extruders," SPE ANTEC Tech. papers, pp. 10-15, 2002.
[3] H. Potente and W. Hanhart, "Design and processing optimization of extruder screws," Polymer Engineering and Science, vol. 34, no. 11, pp. 937-945, 1994.

[4] R. C. Donovan, "Pressure profiles in plasticating extruders," Polymer Engineering and Science, vol. 11, no. 6, pp. 484-491, 1971.

[5] J. G. A. Lovegrove and J. G. Williams, "Pressure generation mechanisms in the feed section of screw extruders," Polymer Engineering and Science, vol. 14, no. 8, pp. 584-594, 1974.

[6] H. Potente and J. Jungemann, "Pressure/throughput behaviour in the melting section," Polymer Engineering and Science, vol. 20, no. 5, pp. 329-350, 2000.

[7] C. Rauwendaal, "Throughput-pressure relationships for power law fluids in single screw extruders," Polymer Engineering and Science, vol. 26, no. 18, pp. 1240-1244, 1986.

[8] C. Rauwendal, Polymer extrusion, 4th ed. Hanser: Munich, 2001, Ch. 4.

[9] C. Rauwendaal, "Extruder output pressure-relationships for power law fluids including leakage flow," SPE ANTEC Tech. papers, p. Paper \# 770, 2002.

[10] Z. Tadmor, S. D. Lipshitz, and R. Lavie, "Dynamic model of a plasticating extruder," Polymer Engineering and Science, vol. 14, no. 2, pp. 112-119, 1974.

[11] M. H. Costin, P. A. Taylor, and J. D. Wright, "On the dynamics and control of a plasticating extruder," Polymer Engineering and Science, vol. 22, no. 17, pp. 1095-1106, 1982.

[12] A. Kochhar and J. Parnaby, "Dynamic modelling and control of plastics extrusion processes," Automatica, vol. 13, no. 2, pp. 177-183, 1977.

[13] L. P. Tan, A. Lotfi, E. Lai, and J. B. Hull, "Soft computing applications in dynamic model identification of polymer extrusion process," Applied Soft Computing, vol. 4, no. 4, pp. 345-355, 2004.

[14] A. J. Botten, A. S. Burbidge, and S. Blackburn, "A model to predict the pressure development in single screw extrusion," Journal of Materials Processing Technology, vol. 135, no. 2-3, pp. 284-290, 2003.

[15] Y. Bereaux, Y. J. Charmeaub, and M. Moguedet, "A simple model of throughput and pressure development for single screw," Journal of Materials Processing Technology, vol. 209, no. 1, pp. 611-618, 2009.

[16] K. Li, J. Peng, and G. W. Irwin, "A fast nonlinear model identification method," IEEE Transactions on Automatic Control, vol. 50, no. 8, pp. 1211-1216, 2005

[17] K. Li, J. Peng, and E. Bai, "A two-stage algorithm for identification of nonlinear dynamic systems," Automatica, vol. 42, no. 7, pp. 11891197, 2006.

[18] Q. M. Zhu and S. A. Billings, "Fast orthogonal identification of nonlinear stochastic models and radial basis function neural networks," International Journal of Control, vol. 64, no. 5, pp. 871-886, 1996.

[19] X. Hong, R. Mitchell, S. Chen, C. J. Harris, K. Li, and G. W. Irwin, "Model selection approaches for non-linear system identification: a review," International Journal of Systems Science, vol. 39, no. 10, pp. 925-946, 2008.

[20] K. Li, J. Peng, and E.-W. Bai, "Two-stage mixed discrete-continuous identification of radial basis function (rbf) neural models for nonlinear systems," IEEE Transactions on Circuits and Systems, vol. 56, no. 3 , pp. 630-643, 2009.

[21] S. Chen, S. Billings, and W. Luo, "Orthogonal least squares methods and their application to non-linear system identification," International Journal of Control, vol. 50, pp. 1873-1896, 1989.

[22] C. Abeykoon, K. Li, M. McAfee, P. J. Martin, Q. Niu, A. L. Kelly, and J. Deng, "A new model based approach for the prediction and optimisation of thermal homogeneity in single screw extrusion," Control Engineering Practice, vol. doi:10.1016/j.conengprac.2011.04.015, 2011.

[23] H. W. Gitschner and J. Lutterback, "Influences of barrel wall temperature on melt throughput fluctuations in the single screw extruder," Kunststoffe, vol. 74, no. 1, pp. 7-8, 1984.

[24] E. C. Brown, A. L. Kelly, and P. D. Coates, "Melt temperature homogeneity in single screw extrusion: effect of material type and screw geometry," SPE ANTEC Tech. papers, pp. 183-187, 2004.

[25] A. L. Kelly, E. C. Brown, and P. D. Coates, "The effect of screw geometry on melt temperature profile in single screw extrusion," Polymer Engineering and Science, vol. 46, no. 12, pp. 1706-1714, 2006.

[26] C. I. Chung, W. J. Hennessey, and M. Tusim, "Frictional behavior of solid polymers on a metal surface at processing conditions," Polymer Engineering and Science, vol. 17, no. 1, pp. 9-20, 1977. 